\title{
Diode pumping of a solid-state laser rod by a two-dimensional CPC-elliptical cavity with intervening optics
}

\author{
Dawei Liang, Rui Pereira * \\ CEFITEC, Departamento de Física, FCT, Universidade Nova de Lisboa, 2825, Campus de Caparica, Portugal
}

Received 5 October 2006; received in revised form 2 February 2007; accepted 21 February 2007

\begin{abstract}
The pump radiation from a large area two-dimensional diode stack is concentrated into a cylindrical intervening optics by a first-stage 2D-CPC concentrator. The compressed radiation from the intervening optics is then efficiently coupled into a laser rod by a second-stage 2D-elliptical pump cavity. Depending on the width of the pump source, optimized rod mounting position is found through a non-sequential ray-tracing analysis. By comparing with the performance of a 2D-CPC-CPC cavity, significant improvements in both absorption efficiency and absorption distribution are achieved by a 2D-CPC-EL cavity. This asymmetric pumping scheme constitutes also an effective alternative to conventional symmetric pumping approaches.
\end{abstract}

(C) 2007 Elsevier B.V. All rights reserved.

Keywords: Lasers; Diode-pumped; Pumping

\section{Introduction}

In the past years the research and studies of highly efficient, high beam quality and long-time operation of solidstate lasers pumped by diode lasers instead of discharge lamps have been maintained by the performance improvements of semi-conductor diode-lasers arrays. High efficiency can be obtained because there is a good spectral match between the emission spectrum of the pump beam and the absorption spectrum of the laser medium. The most efficient laser systems have end-pumping configurations. The reason for the increased efficiency of the endpumping is that the pump power is absorbed over a small centred region within the laser rod leading to a much higher inversion density than in side-pumping, where a sizable amount of the pump power is absorbed near the surface, not in the centre, of the gain medium. The highest inversion density in a region the same size or smaller than the fundamental mode is the deciding factor for low thresh-

\footnotetext{
* Corresponding author. Tel.: +351 212948576; fax: +351 212948549.

E-mail addresses: daweiliang@clix.pt (D. Liang),r.p.pereira@gmail. com (R. Pereira).
}

old and high optical efficiency [1,2]. For cylindrical gain media, the end-pumping and close-coupled side-pumping geometries approach the ideal $\mathrm{TEM}_{00}$ mode-matched absorption distribution, but they have total pump-power limitations. Side-pumping is a simpler configuration for power scaling as it gives uniform absorption along the axis of the laser rod and spreads the absorbed power over a large area, thus reducing the thermal loading. The pump radiation from the diode stacks can be compressed by either compound parabolic concentrators or by wedge lenses into the diffusive cavity chamber surrounding the laser rod. The rod absorbs the pump light and converts it into infrared laser light [3-6]. However these methods do not allow for flexibility in shaping the pump beam distribution inside the medium, leading to nearly homogeneous output profiles. To tailor the pump distribution within the medium, the micro-lensed diode stacks $[7,8]$ are required since the concentration ratio is limited by the divergence of the pump beams. The typical transfer efficiency of a commercial micro-lens is about $85 \%$, which has been one of the reasons for low wall-plug efficiency. Inserting optics, such as optical fibres, between the diode laser and the pump cavity increases the laser head complexity. 
However, the pump-beam distribution can peak at the centre of the medium [9], resulting in an improved match of the pump distribution with the resonator mode. For lowaverage-power applications, in which thermal lensing is moderate, the overlap of the laser mode with an excitation peaked at the centre of the rod can be advantageous.

The most suitable pump cavity is the one that efficiently concentrates the radiation from many diode lasers into solid-state gain medium when the diodes are not densely packed and are placed over a large entry aperture [10]. By sparsely packing the diodes, we can reduce thermal loading problems in the array and therefore wavelengthemission problems. A possible candidate for a pump cavity that can efficiently transfer radiation from an extended, sparsely packed, two-dimensional diode array (ESDA) to the gain medium is a non-imaging concentrator [11]. Although the non-imaging pump cavity provides a large amount of pump power, it does not give a Gaussian absorption profile. What we believe to be a novel pumping scheme is reported in this paper. Symmetric multi-sidepumping arrangements for high-average-power applications are firstly discussed, providing excellent examples for the proposed asymmetric 2D-CPC-EL pumping scheme. Having nearly the same effectiveness as conventional symmetric schemes, the 2D-CPC-EL proposal constitutes an alternative approach in dealing with the radiation-coupling problem from a large area two-dimensional micro-lens-free diode stack into a cylindrical laser medium. Non-sequential ray-tracing is performed to analyze both the absorption efficiencies and the absorption distributions within the Nd:YAG laser rods of different diameters. By comparing with the performances of the 2D-CPC-CPC cavity, the superiority of the 2D-CPC-EL cavity with intervening optics is confirmed, leading to a much stronger pump radiation concentration within the small centred region of the rod. This scheme can provide better matching between the lasing mode volume and the pump volume generated due to the focusing properties of both the truncated elliptical pump cavity and the cylindrical intervening optics.

\section{High-average-power symmetrical multi-side-pumping arrangement}

For low-average-power applications such as laser oscillators, an excellent efficiency of pump absorption $(>80 \%$ overall), a good pump symmetry around the rod and the required peak on-axis to enhance low order modes are three important features of a modern diode-pumped solid-state laser with symmetric pumping arrangement. A limited number of horizontal or vertical diode arrays can provide sufficient pump power for the efficient production of high quality laser beam.

For high-average-power applications such as laser amplifiers, a huge amount of pump power can be obtained from either a large area diode stack in rectangular arrangement or from many narrow vertical diode stacks in several- folded pump cavity segments. Fig. 1 shows the schematic drawing of a symmetric pump cavity composed of 16 narrow vertical diode stacks, distributed uniformly around cylindrical surface with $80 \mathrm{~mm}$ perimeter and $100 \mathrm{~mm}$ height. The laser rod of $6.35 \mathrm{~mm}$ diameter and $115 \mathrm{~mm}$ length is water-cooled within the flow tube of $11 \mathrm{~mm}$ external diameter.

In Fig. 1, each diode laser emitter in each of the 16 vertical diode stacks has an elliptical Gaussian emission with FWHM $12^{\circ}$ divergence in the slow-axis direction and FWHM $40^{\circ}$ in the fast-axis direction. The fast-axis of the diode stacks are arranged parallel to the laser rod axis. Therefore the pump beams are incident upon the laser medium in $\mathrm{P}$ polarization, which increases the transmittance at the rod surface and leads to high pump efficiency. In the fast-axis direction, the pump radiations reach the rod either directly or by the reflections from two end plates (not shown in Fig. 1). The fast-axis divergence of $40^{\circ}$ also assures to filling more uniformly the rod along the optical axis while the $12^{\circ}$ slow axis divergence enables an easy direct light coupling from the diode stack to the laser rod through the flow tube with cooling water. For easy comparison, $1000 \mathrm{~W}$ is considered as the total power from the pump source in each case of this paper.

The peak absorption at $808 \mathrm{~nm}$ is nearly 10 per $\mathrm{cm}$ for a $\mathrm{Nd}$ :YAG laser medium with $1.0 \%$ doping. To reach sufficient absorption of pump power, one has to control the emitted wavelength of the diode stacks. For low-averagepower applications, tuning to the peak absorption can however cause side-lobes at the input surface of the rod [6]. At high-average-power, the laser rod of lower absorption coefficient $(0.6 \%$ doping, for example) is usually used to achieve a uniform absorption distribution. The absorption coefficient of 4 per $\mathrm{cm}$ is therefore assumed as a representative average value. The laser rod of $6.35 \mathrm{~mm}$ diameter is water-cooled within the anti-reflection-coated flow tube of $11 \mathrm{~mm}$ external and $9 \mathrm{~mm}$ internal diameters. The side-surface of the laser rod is modelled as uncoated. Actual ray propagation within the crystal rod is threedimensional. However, the number of rays that propagate

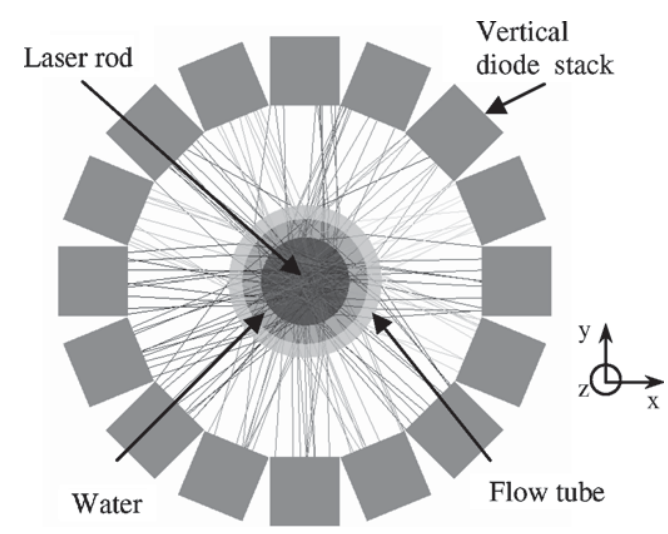

Fig. 1. Sixteen-side-puming arrangement for high-average-power applications. 
in $\pm z$ direction can be assumed to be equal on average and the ray distribution in the $\pm z$ direction is uniform as a result. For this reason, we consider only the ray distribution within two-dimensional cross-section perpendicular to the $z$ direction. The cross-sectional area of the laser crystal is made up of 10,000 zones. During ray-tracing, the path length in each intercepted zone is found. With this value and the absorption coefficient of 4 per $\mathrm{cm}$ for $\mathrm{Nd}$ :YAG material, the power absorbed by both the laser rod and by the cylindrical volumes of $2 \mathrm{~mm}$ and $1 \mathrm{~mm}$ diameters in the central core regions can be calculated correspondingly, as shown in Tables 1-3. Similarly, the analysis of the $4 \mathrm{~mm}$ diameter rod is carried out. Due to reduced absorption of the pump radiation near the rod surface, more pump radiation is absorbed within the central core region of the $4 \mathrm{~mm}$ diameter rod. On the other hand, small diameter rod intercepts less incident pump radiation, lowering hence its overall absorption efficiency.

Sixteen narrow vertical diode stacks are used to achieve an excellent absorption distribution as demonstrated in Fig. 2. Each diode stack is $5 \mathrm{~mm}$ in width and $100 \mathrm{~mm}$ in length. Table 1 lists the total absorption efficiencies by the laser rods of $6.35 \mathrm{~mm}$ and $4.0 \mathrm{~mm}$ diameters. The absorption efficiencies within the cylindrical volumes of $1 \mathrm{~mm}$ and $2 \mathrm{~mm}$ diameters are given. When the vertical laser diode stacks of $10 \mathrm{~mm}$ widths are used for pumping directly the rod, an 8-side-pumping scheme is formed. Both the pump radiation absorption efficiency and absorption

Table 1

Pump radiation absorption efficiencies for the 16-side-pumping arrangement

\begin{tabular}{llll}
\hline $\begin{array}{l}\text { Rod } \\
\text { diameter } \\
(\mathrm{mm})\end{array}$ & $\begin{array}{l}\text { Absorption } \\
\text { efficiency }(\%)\end{array}$ & $\begin{array}{l}\text { Within } \varnothing 2 \mathrm{~mm} \\
\text { cylindrical volume } \\
(\%)\end{array}$ & $\begin{array}{l}\text { Within Ø1 mm } \\
\text { cylindrical volume } \\
(\%)\end{array}$ \\
\hline 6.35 & 85.00 & 11.60 & 3.06 \\
4.0 & 59.60 & 18.38 & 4.80 \\
\hline
\end{tabular}

Table 2

Pump radiation absorption efficiencies for 8-side-pumping arrangement

\begin{tabular}{llll}
\hline $\begin{array}{l}\text { Rod } \\
\text { diameter } \\
(\mathrm{mm})\end{array}$ & $\begin{array}{l}\text { Absorption } \\
\text { efficiency }(\%)\end{array}$ & $\begin{array}{l}\text { Within } \varnothing 2 \mathrm{~mm} \\
\text { cylindrical volume } \\
(\%)\end{array}$ & $\begin{array}{l}\text { Within } \varnothing 1 \mathrm{~mm} \\
\text { cylindrical volume } \\
(\%)\end{array}$ \\
\hline 6.35 & 68.10 & 6.13 & 1.54 \\
4.0 & 37.80 & 10.10 & 2.52 \\
\hline
\end{tabular}

Table 3

Pump radiation absorption efficiencies for the 8-side-pumping scheme by using coupling optics

\begin{tabular}{llll}
\hline $\begin{array}{l}\text { Rod } \\
\text { diameter } \\
(\mathrm{mm})\end{array}$ & $\begin{array}{l}\text { Absorption } \\
\text { efficiency }(\%)\end{array}$ & $\begin{array}{l}\text { Within Ø2 mm } \\
\text { cylindrical volume } \\
(\%)\end{array}$ & $\begin{array}{l}\text { Within Ø1 mm } \\
\text { cylindrical volume } \\
(\%)\end{array}$ \\
\hline 6.35 & 85.13 & 12.40 & 3.33 \\
4.0 & 64.23 & 20.4 & 5.31 \\
\hline
\end{tabular}

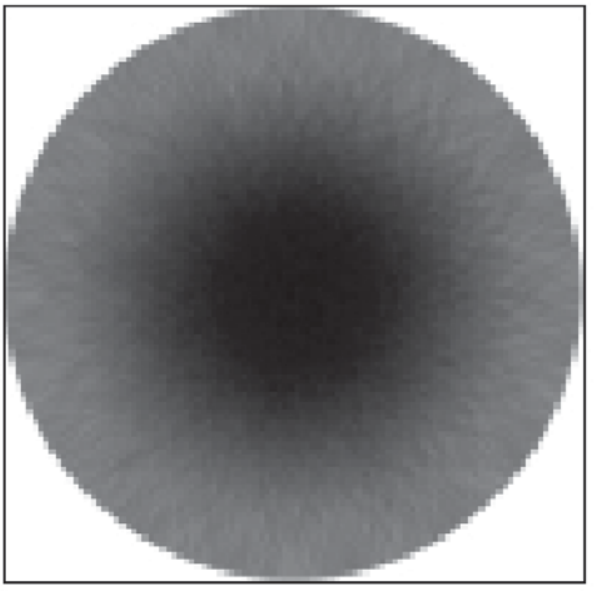

1.3293

1.1963

I. . 0634

0.9305

ㅁ. 7976

0.6646

0.5317

(1. 3998

ㅈ. 365무

日. 1329

(6. . .000

Fig. 2. Grey-scale absorption distribution for the 16-side-pumping arrangement. Black signifies near or at maximum absorption for this plot whereas white signifies little or no absorption. (vertical unit in $\mathrm{W} / \mathrm{mm}^{2}$ ).

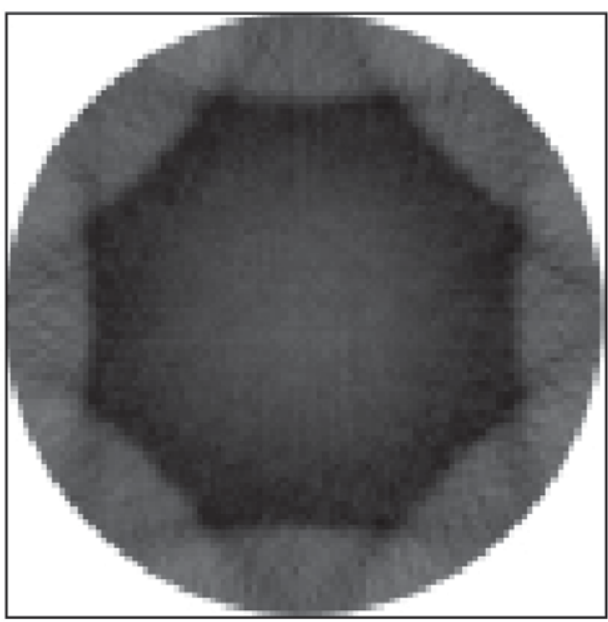

(0.7748

0.6973

0.6 6198

6. 5423

口. 4645

0. 3974

0,3099

0.3934

G. $16 E 0$

6. . 0775

(10.000

Fig. 3. Grey-scale absorption distribution for the 8-side-pumping arrangement (vertical unit in $\mathrm{W} / \mathrm{mm}^{2}$ ).

distribution degrade rapidly, as is easily noticeable by comparing Table 1 with Table 2 and Fig. 2 with Fig. 3.

Coupling optics is therefore necessary to concentrate more pump radiations into the laser rod. Two cylindrical lenses are combined to couple individually the pump radiation from each diode stack to the rod. There are 16 cylindrical lenses mounted between the flow tube and eight diode stacks, each with $10 \mathrm{~mm}$ width. The absorption distribution peaks along the central core region of the rod. Slightly higher absorption efficiencies are achieved by using coupling optics, as shown in Table 3.

The symmetric side-pumping arrangement has the following advantages:

1. For low-average-power applications, the most widely used symmetric side pumping arrangement combines both an excellent efficiency of pump absorption with a superb absorption distribution. The required peak on- 
axis distribution is ensured. The laser rod is normally pumped symmetrically by only several laser diode bars or diode-stacks, this is a natural configuration and is very difficult to challenge.

2. For high-average-power applications, the symmetric and direct pumping of laser rod by many narrow vertical diode stacks of limited widths (usually several millimetres) can ensure simultaneously both a high coupling efficiency and an excellent absorption distribution.

The symmetric multi-side-pumping arrangement suffers from the following drawbacks:

1. The pump radiation absorption efficiency and the gain distribution degrade rapidly with the increase of the width of each vertical diode stack. To the best of our knowledge, the vertical diode stacks of only several millimetres in widths are still not commercially available. The mounting and cooling of so many arrow diode stacks will be mechanically difficult. Adding coupling optics individually to each of the each diode stack can concentrate more pump radiation within the laser medium. The pumping scheme becomes, however, even much more complex.

2. To reach sufficient absorption of the pump power, one has to control the emitted wavelength of the stacks by the adequate temperature control of heat sinks. As the number of diode stacks increases, it becomes increasingly difficult to tune simultaneously all these diode stacks.

The combination of two-dimensional diode laser-stacking concept with asymmetric rod-pumping concept can, in our opinion, avoid the disadvantages mentioned above. For low-average-power applications, an extended, sparsely packed, two-dimensional diode array (ESDA) can be used as the pump source to reduce thermal loading problems in both laser rod, because of the wavelength matching of the source-emission band to the solid-state absorption band, and in the diode array, because of the sparse packing of individual diodes. For high-average-power applications, the commercially available two-dimensional diode stacks integrate multiple actively or passively cooled diode laser bars on massively mounts. Back-plane cooling of the stack element allows nearly unlimited diode bar stacking. Extremely powerful diode laser pump source can therefore be achieved. Both the wavelength control and the mechanical mounting of these commercially available products become much easier than conventional symmetrical approaches.

With respect to pumping a rod-geometry medium, diode stacks require radiation control because of the geometrical mismatch between the laser medium and the extended light emitting area of the pump source. The coupling of pump radiation from a large area two-dimensional micro-lensfree diode stack to a cylindrical gain medium is an important technical challenge which deserves further researches. It is based on this idea that the double-stage 2D-CPCEL pumping scheme is proposed.

\section{Double-stage 2D-CPC-CPC and double-stage 2D-CPC-EL pump cavities}

\subsection{Single-stage and double-stage CPC pump cavities}

For a simple system involving only a large area laser diode pump source, a gain medium and a reflector, a single-stage compound parabolic concentrator (CPC) is commonly designed by observing the edge-ray principle of non-imaging optics [12]. The CPC is used to convert the radiation from a large-area homogenous source emitting uniformly into a small angle, to a small-area homogenous output emitting uniformly into a large angle, thus the source étendue is preserved. This preservation implies that irradiance is larger at the output surface than at the entrance aperture, leading to a net concentration of the pump radiation. Edge-ray design methods are however too constrained to provide the desired absorption distribution over a volume within a single-stage CPC. There are nevertheless numerous applications of when the absorption distribution within the volume is beneficial in laser technology. A double-stage CPC-CPC type concentrator was used in the Sun-pumped laser experiment [13]. The high pump density system was composed of a three-dimensional CPC with $98 \mathrm{~mm}$ entrance diameter and $24 \mathrm{~mm}$ exit diameter, followed by a two-dimensional CPC with $24 \times 33 \mathrm{~mm}$ rectangular aperture. It pumped a $6 \mathrm{~mm}$ diameter $72 \mathrm{~mm}$ long Nd:YAG laser rod, which emitted up to $45 \mathrm{~W}$ laser power, resulting in $6.7 \mathrm{~W} / \mathrm{m}^{2}$ laser efficiency. The large $M^{2}$ factor was however unsatisfactory. It is therefore desirable to search for a different cavity design that concentrates more pump radiation within the central core region of the laser rod.

\subsection{Double-stage 2D-CPC-EL pump cavity with cylindrical intervening optics}

A lamp-pumped laser makes use of a hollow reflective pump cavity such as an elliptical chamber to couple the radiation from the radiant source into the laser rod. The elliptical cavity is based on the geometrical theorem that rays originating from one focal line of an elliptical cylinder are reflected to the other focal line; therefore, the energy can be transferred from a line source (arc lamp) to a line absorber (laser crystal). As a consequence of the preservation of angles, the portion of the elliptical reflector nearer the lamp forms a magnified image at the laser rod while that portion nearer the crystal forms a reduced image of the lamp. For an asymmetrical diode-side-pumped laser with a truncated elliptical chamber, only the portion of the elliptical reflector nearer the crystal is used, resulting therefore in a reduced (focused) image within the laser rod. The transfer efficiency of the elliptical cavity and its dependence upon the cross-sectional dimension of the reflector, the radiant source, the laser crystal, the wall reflectivity and the ellipse eccentricity were extensively discussed in early works [14-17]. Besides the optical coupling 
efficiency, the pump cavity also determines the pump light distribution within the laser crystal [18].

The double-stage 2D-CPC-EL pump cavity consists of a large first-stage two-dimensional CPC concentrator followed by a small second-stage two-dimensional elliptical pump cavity, as shown in Figs. 4a and 5a.

Similar to the symmetrical side pumping scheme discussed in previous section, the large area rectangular pump source is oriented such that the fast-axis direction is along the axis of the laser rod and the slow-axis direction is perpendicular to it. The pump radiations reach the rod by reflections within the pump cavity. The fast-axis divergence of $40^{\circ}$ also assures to filling more uniformly the rod along the optical axis while the $12^{\circ}$ slow-axis divergence facilitates the efficient light concentration to the laser rod by the 2D-CPC-EL cavity.

The rectangular light emitting area of pump source can occupy either the whole or a part of the entire entry aperture $(100 \times 100 \mathrm{~mm})$ of the first-stage 2D-CPC. The firststage CPC profile is designed by observing the edge-ray principle of non-imaging optics [12]. The optical invariant equation $d_{1} \sin \theta_{1}=d_{2} \sin \theta_{2}$ relates the important parameters of the first-stage concentrator, where $d_{1}$ is its entrance aperture of $100 \mathrm{~mm}$ in $x$ direction, $\theta_{1}$ is the half-angle of the input beam divergence of $11^{\circ}$ (encompassing $98 \%$ radi-
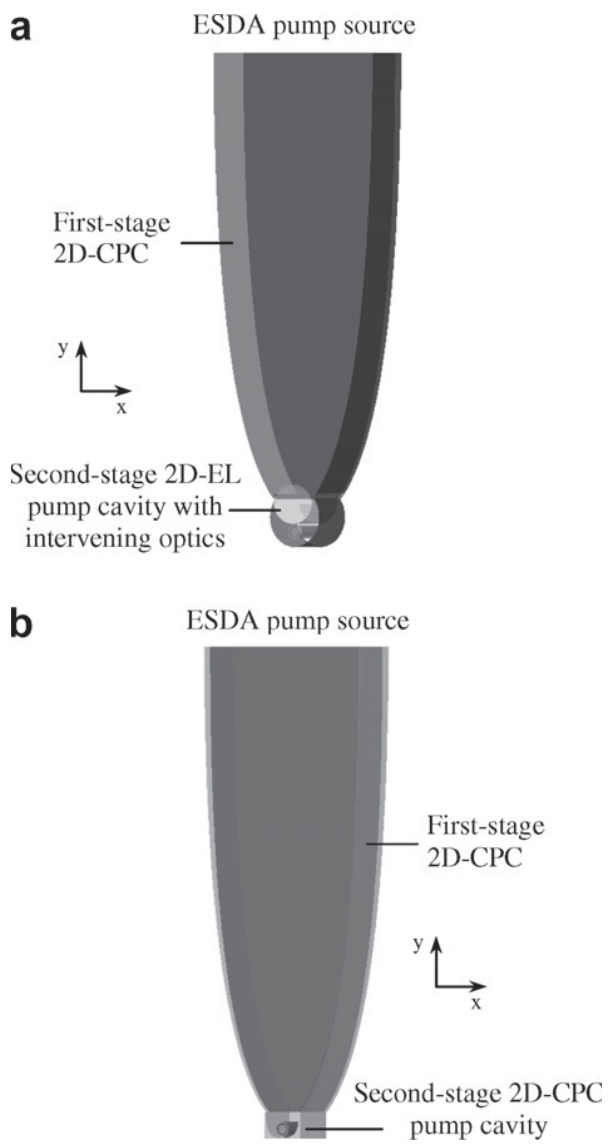

Fig. 4. Three-dimensional views of the 2D-CPC-EL and the 2D-CPCCPC pump cavities.
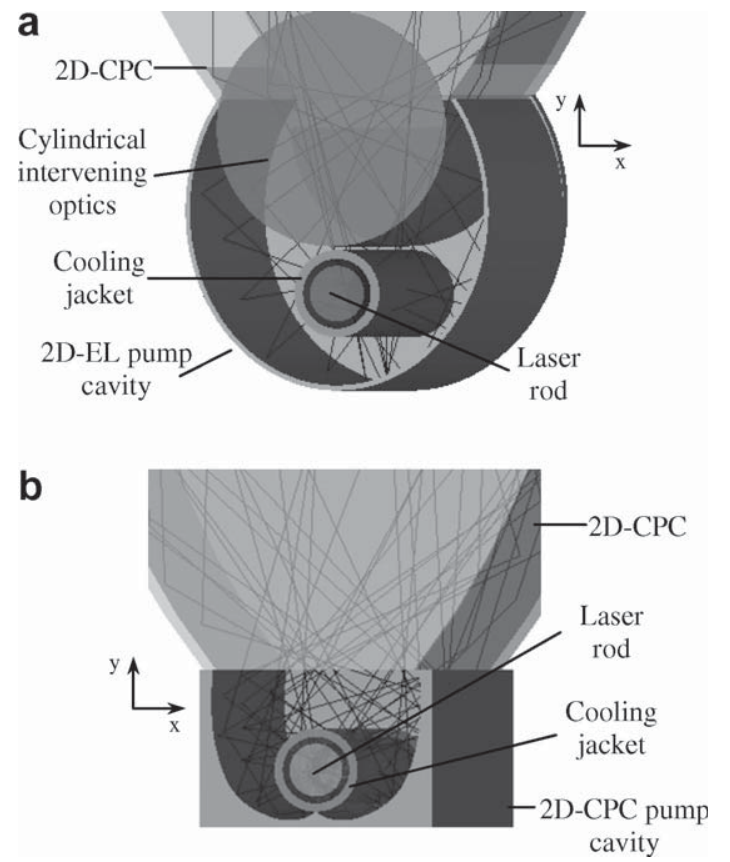

Fig. 5. Detailed partial three-dimensional views of the 2D-CPC-EL and the $2 \mathrm{D}-\mathrm{CPC}-\mathrm{CPC}$ pump cavities (only 10 trace rays are represented in each figure).

ation of the two-dimensional pump source), $d_{2}$ is its exit aperture (of $24 \mathrm{~mm}$ ) in $x$ direction, and $\theta_{2}$ is the half angle of the exit beam divergence of $55^{\circ}$. Extruding this crosssectional profile along the length of the laser rod forms the 2D-CPC cavity. The first-stage CPC funnels the pump radiation out of its $24 \times 100 \mathrm{~mm}$ rectangular exit aperture at $110^{\circ}$ full angle. The 2D-CPC is $300 \mathrm{~mm}$ in height and can be further truncated without largely affecting the efficiency performance.

The concentrated light from the first-stage 2D-CPC enters the $24 \times 100 \mathrm{~mm}$ aperture of the truncated elliptical pump cavity through cylindrical intervening optics (a fused silica cylinder $26 \mathrm{~mm}$ in diameter and $100 \mathrm{~mm}$ in length positioned parallel to the $11 \mathrm{~mm}$ diameter cooling jacket). The pump radiation is then efficiently coupled into a $6.35 \mathrm{~mm}$ diameter Nd:YAG rod by a truncated two-dimensional elliptical (2D-EL) pump cavity. The focusing abilities of both the truncated elliptical cavity $(a=20 \mathrm{~mm}$, $e=0.4$, truncated height $=8 \mathrm{~mm}$ and cavity length $=$ $100 \mathrm{~mm}$ ) and the cylindrical intervening optics ensure the strong pump light concentration within the central core volume of the rod. Depending on the specific width of the rectangular pump source, optimum laser rod mounting position can be determined. The first-stage 2D-CPC and the second-stage 2D-EL pump cavity are water-cooled, each in a separate flow loop.

\subsection{Double-stage 2D-CPC-CPC pump cavity}

The double-stage 2D-CPC-CPC pump cavity is also composed of a large first-stage 2D-CPC concentrator fol- 
lowed by a small second-stage 2D-CPC pump cavity, as shown in Figs. 4b and 5b. To compare correctly both the absorption efficiencies and the absorption distributions of the laser rod, pumped by either the 2D-CPC-EL or the 2D-CPC-CPC cavity, it is indispensable to use the same large area two-dimensional pumping source and the same first-stage 2D-CPC concentrator. After being concentrated by the same first-stage $2 \mathrm{D}-\mathrm{CPC}$, the pump radiation reaches finally the $24 \times 100 \mathrm{~mm}$ entry aperture of a second-stage 2D-CPC pump cavity. The cross-section shape of this cavity is designed with two variables: the acceptance angle of the concentrator and the radius of the laser rod. The rod diameter of $6.35 \mathrm{~mm}$ is determined by equation [12]: $d_{1} \sin \theta_{1}=\pi d$, where $d_{1}$ is the input aperture of $24 \mathrm{~mm}$ in $x$ direction and $\theta_{1}$ is the input beam acceptance half-angle of $56^{\circ}$, which encompasses $98 \%$ of incident pump radiation and is slightly larger than output semiangle of $55^{\circ}$ from the first-stage CPC concentrator. $d$ is the laser rod diameter. Extruding this cross-section along the length of the laser rod forms a theoretical 2D-CPC pump cavity. The practical second-stage $2 \mathrm{D}-\mathrm{CPC}$ pump cavity is designed by slightly over sizing the theoretical CPC reflector to adapt the flow tube [19]. This design still preserves the ideal flux concentration around the absorber. The laser rod is mounted inside the quartz flow tube and is water-cooled, as is the second-stage 2D-CPC pump cavity, each in a separate flow loop.

\section{Absorption efficiency and absorption distribution improvements by the 2D-CPC-EL cavity with cylindrical intervening optics}

\subsection{Non-sequential ray-tracing code}

For maximizing the pump radiation within the laser rod under different pumping conditions, namely, the width variation of the rectangular pump source, ZEMAX nonsequential ray-tracing study is carried out to discover the optimized mounting position for the laser rod within the truncated elliptical pump cavity. Maximum absorption efficiency and optimum absorption distribution can subsequently be analyzed. The optimization of the variables and hence the improvement in the pump cavity performance is based upon the following design considerations. Each diode laser emitter in the full 2-D pump source has an elliptical Gaussian emission with FWHM $12^{\circ}$ divergence in the slow-axis direction and FWHM $40^{\circ}$ in the fast-axis direction. Each laser diode is linearly polarized in $\mathrm{P}$ plane and relatively low reflectance is observed at large incident angle in relation to the normal line of the inner surface of both the first-stage concentrator and the second-stage pump cavity. The reflectance recuperates however to nearly $100 \%$ at the grazing incident angles of nearly $90^{\circ}$. The transmittance at the surfaces of the cylindrical intervening optics, the flow tube and the laser rod, on the other hand, is enhanced due to the use of P-polarized pump radiation. For pump radiation polarized in $\mathrm{P}$ plane, once the metallic and the dielectric coating files are defined by considering both the reflectance and the transmittance at different incident angles ranging from 0 to $90^{\circ}$, the ray tracing can be conducted correctly. All reflectors in this study are smooth, specular and $99 \%$ reflectivity at normal incidence is assumed. All the optical components such as the cylindrical intervening optics and the cooling jacket are anti-reflection coated. No phase information is retained for the diode radiation, thus an incoherent ray tracing model can be used. The two-dimensional pump cavity with truncated elliptical cross-section $(a=20 \mathrm{~mm}$, $e=0.4$, truncated height $=8 \mathrm{~mm}$, cavity length $=100 \mathrm{~mm}$ ) is found to be the optimum parameters to start the raytracing analysis. The side-surface of the laser rod of $115 \mathrm{~mm}$ length is modelled as uncoated.

In ray-tracing analysis, the cylindrical $6.35 \mathrm{~mm}$ diameter gain medium is divided into a total of 10,000 zones. During ray-tracing, the path length in each intercepted zone is found. With this value and the effective absorption coefficient (4 per $\mathrm{cm}$ ) of the Nd:YAG material, the power absorbed within the central cylindrical volumes can be calculated by summing up the absorbed pump radiations of all the zones within theses volumes. Total absorbed radiation of $6.35 \mathrm{~mm}$ rod is also calculated. For each of the rectangular pump source with its specific width, non-sequential ray-tracing is carried out to localize the optimum mounting position for the laser rod within the elliptical cavity, where the highest pump absorptions within the central cylindrical volumes of $1 \mathrm{~mm}$ and $2 \mathrm{~mm}$ diameters serve as our design criteria. Optimized mounting lines with $+0.2 \mathrm{~mm}$, $-0.3 \mathrm{~mm}$ and $-0.9 \mathrm{~mm}$ offsets relative and parallel to the second focal line of the elliptical pump cavity are found, each corresponding to one of the widths $L=60 \mathrm{~mm}$, $80 \mathrm{~mm}$ or $100 \mathrm{~mm}$ of the rectangular pump source.

\subsection{Absorption efficiency and absorption distribution (both radial and angular) improvements by the $2 D-C P C-E L$ cavity with cylindrical intervening optics}

The Grey-scale absorption distributions for $6.35 \mathrm{~mm}$ and $4.0 \mathrm{~mm} \mathrm{Nd}$ :YAG rods, pumped, respectively, by the 2-D ESDA source of $L=60 \mathrm{~mm}, 80 \mathrm{~mm}$ or $100 \mathrm{~mm}$ width, are given by Figs. 6-11. Black signifies near maximum absorption for these plots, whereas white signifies little or no absorption. The absorption distributions are improved by the 2D-CPC-EL cavity with cylindrical intervening optics, focusing more radiation into the central core region of the rod. The radial absorption improvements are clearly presented by the absorbed flux/volume distributions along both the central cross-section row and the central cross-section column of the rods, as shown in Figs. 6, 8 and 10. The absorbed flux peaks along the central core region of the rod, favouring the generation of more $\mathrm{TEM}_{00}$ laser power. The 2D-CPC-CPC cavity does not provide a near-Gaussian absorption profile. There exists low absorption density within the central core region. The ray tracing analysis indicates that there is also a strong lack of rotational 
symmetry and the maximum occurs on the sides of the rod. The radial absorption curves pumped by the $2 \mathrm{D}-\mathrm{CPC}-$ CPC cavity are represented by the absorbed flux/volume distributions along both the central cross-section row and the central cross-section column of the rods, as shown in Figs. 7, 9 and 11, indicating the lack of absorbed flux in the central rod region. The $2 \mathrm{D}-\mathrm{CPC}-\mathrm{CPC}$ cavity is designed according to the edge-ray principle, prejudicing therefore the concentration of pump power in the central core region.

When pumped by the 2D-CPC-EL cavity, the average absorbed flux/volume values in Figs. 6, 8 and 10 are significantly higher than those values by the 2D-CPC-CPC cavity in Figs. 7, 9 and 11. The absorption efficiencies within $6.35 \mathrm{~mm}$ and $4.0 \mathrm{~mm}$ rods and also within the central core volumes of $2.0 \mathrm{~mm}$ and $1.0 \mathrm{~mm}$ diameters are listed in Tables 4-6. For the 2D-CPC-EL cavity, the absorption efficiency decreases slowly as the width of the pump source increases, while it remains nearly constant for the $2 \mathrm{D}$ -
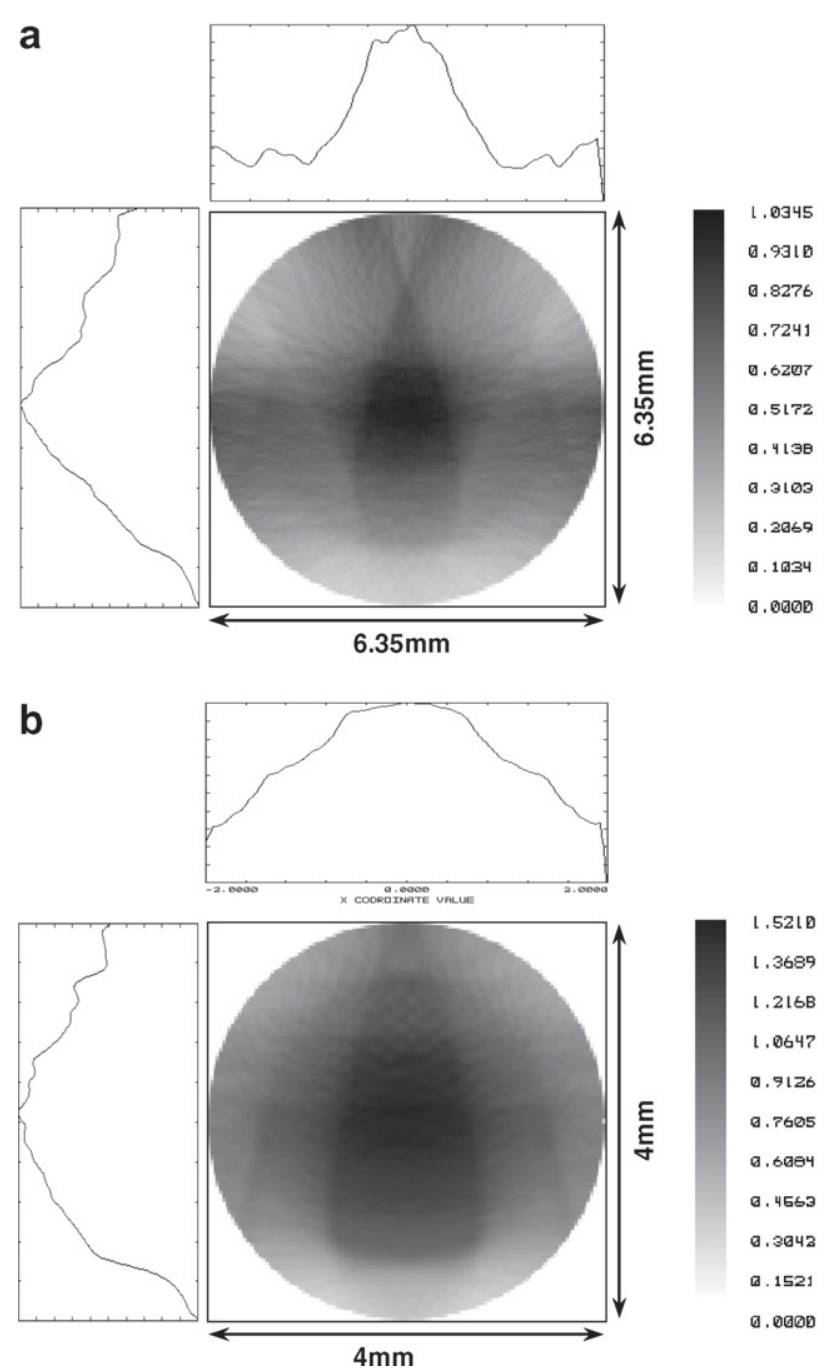

Fig. 6. Grey-scale absorption distribution within the 2D-CPC-EL cavity pumped by the rectangular source of $60 \mathrm{~mm}$ width for (a) $6.35 \mathrm{~mm}$ diameter (b) $4.0 \mathrm{~mm}$ diameter $\mathrm{Nd}$ :YAG rods (vertical unit in $\mathrm{W} / \mathrm{mm}^{2}$ ).
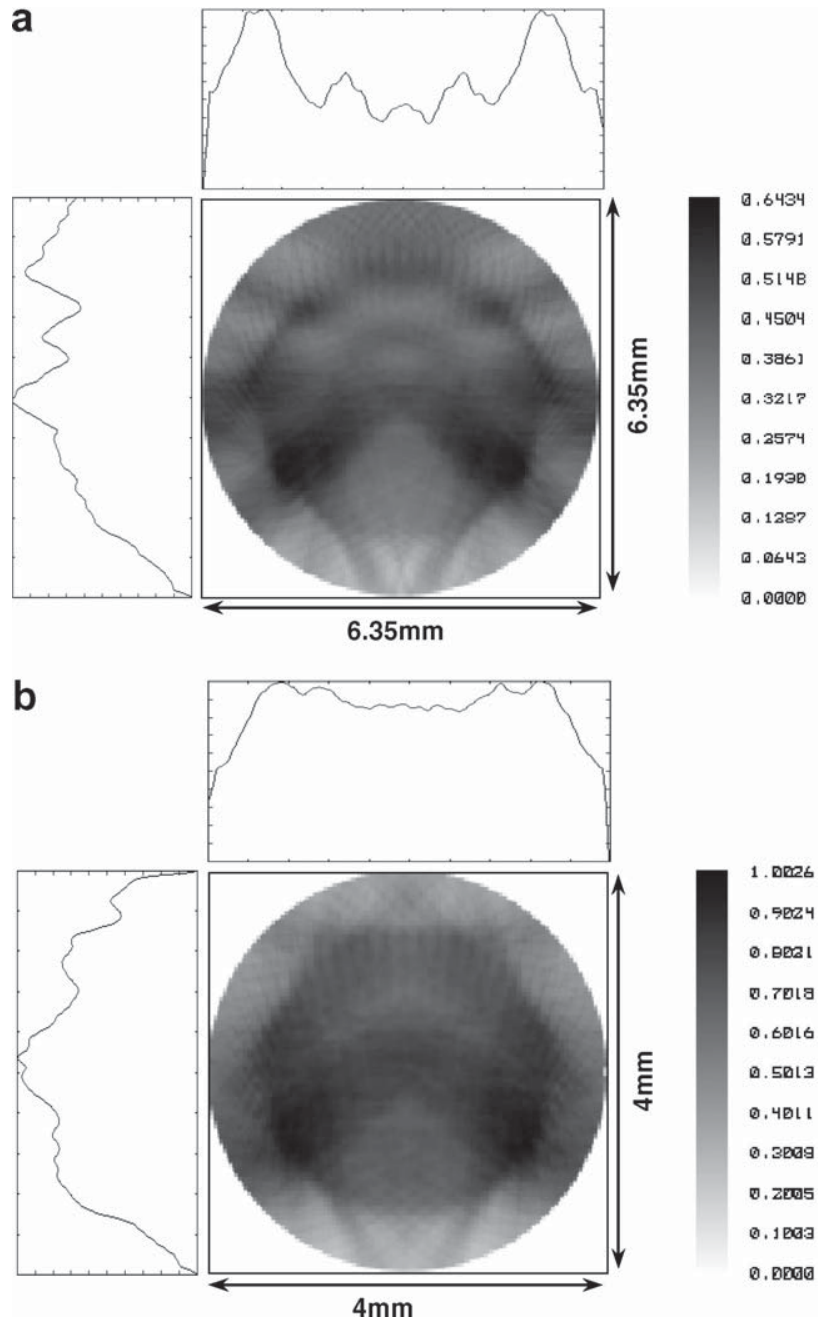

Fig. 7. Grey-scale absorption distribution within the 2D-CPC-CPC cavity pumped by the rectangular source of $60 \mathrm{~mm}$ width for (a) $6.35 \mathrm{~mm}$ diameter (b) $4.0 \mathrm{~mm}$ diameter Nd:YAG rods (vertical unit in $\mathrm{W} / \mathrm{mm}^{2}$ ).

CPC-CPC cavity. There exists an obvious absorption efficiency improvement when the laser rod is pumped by the 2D-CPC-EL cavity.

It is also interesting to notice that the general superiority of the 2D-CPC-EL cavity in achieving high absorption efficiencies shown in Tables 4-6 is surpassed only once by the 2D-CPC-CPC cavity in Table 6, where the non-imaging cavity attains $78.30 \%$ absorption efficiency, higher than $77.42 \%$ by the $2 \mathrm{D}-\mathrm{CPC}-\mathrm{EL}$ cavity. This slight advantage in efficiency is valid only when the maximum pumping width of $L=100 \mathrm{~mm}$ and the maximum rod diameter of $D=6.35 \mathrm{~mm}$ are considered in the ray tracing analysis. This result is in conformity with the maximum light concentration principle by CPC in non-imaging optics.

Unlike $6.35 \mathrm{~mm}$ rod where a sizable amount of the pump power is absorbed near the surface, not in the centre of the crystal, the use of $4 \mathrm{~mm}$ rod greatly enhances the absorption in the centre region of the rod. High pumping density or small rod surface is attractive but challenging in solid-laser research. With small rod diameter, high-order 

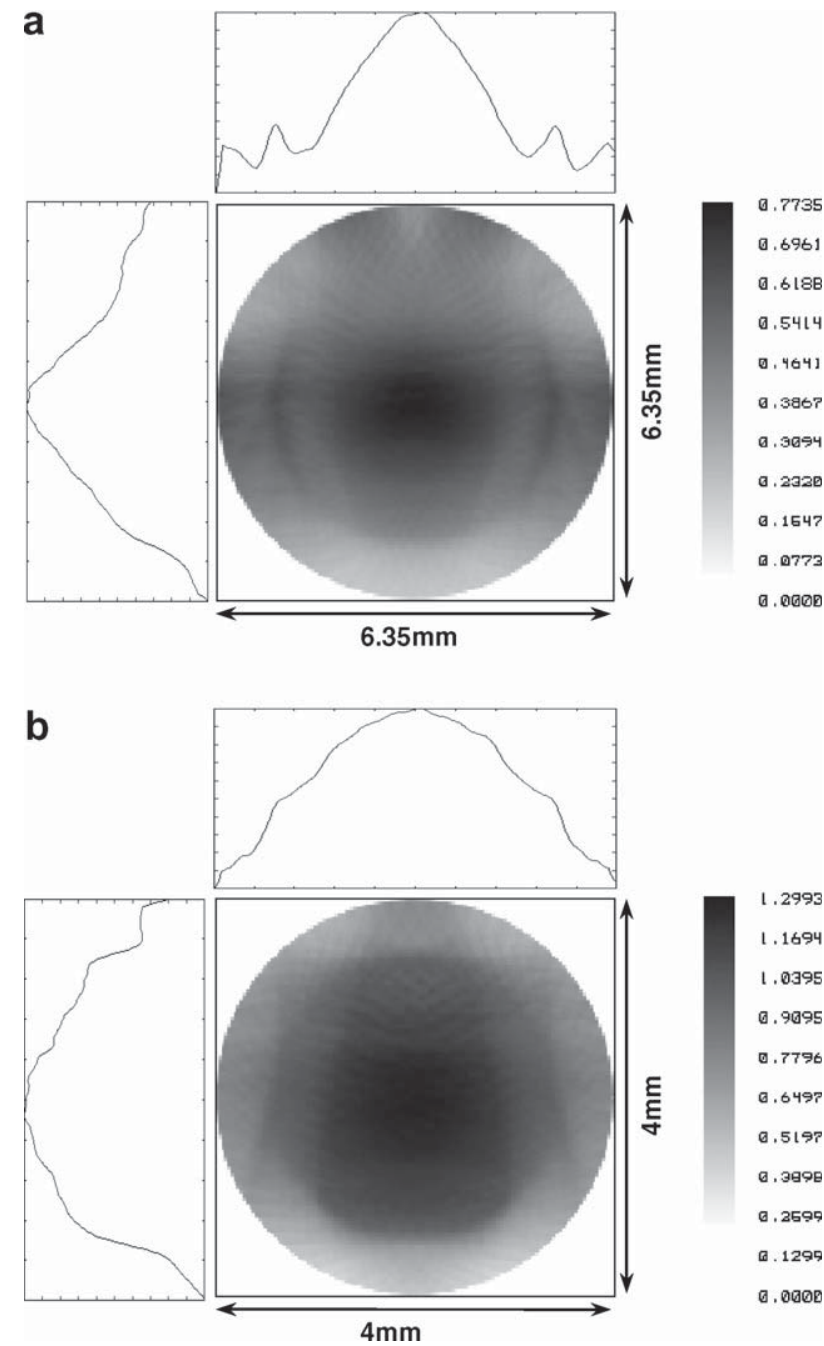

Fig. 8. Grey-scale absorption distribution within the 2D-CPC-EL cavity pumped by the rectangular source of $80 \mathrm{~mm}$ width for (a) $6.35 \mathrm{~mm}$ diameter (b) $4.0 \mathrm{~mm}$ diameter Nd:YAG rods (vertical unit in $\mathrm{W} / \mathrm{mm}^{2}$ ).

resonator modes are suppressed by large diffraction losses and beam quality improves. The absorption efficiency enhancement achieved by pumping small-diameter rod through the 2D-CPC-EL cavity will contribute largely to the production of high quality laser beam.

A known problem in Nd:YAG pump calculations is that the diode spectrum from bars is usually $3 \mathrm{~nm}$ wide and tunes significantly with diode current. Depending on the diode heat sink arrangement, as well as temperature - so there is significant variability of the effective absorption. For what is effectively a single-pass pump configuration, there will be significant variation in the absorption efficiency and the absorption profile for the Nd:YAG medium. The absorption coefficients of 10 and 6 per $\mathrm{cm}$ are used to investigate the possible scale of the tuning effects and the validity of our conclusions about the 2D-CPC-EL pump cavity. The pump absorptions efficiencies and absorption distributions of the $6.35 \mathrm{~mm}$ rod pumped by the 16-side symmetric approach, the 2D-CPC-EL and the 2D-CPCCPC asymmetric schemes are analysed by ZEMAX ray-
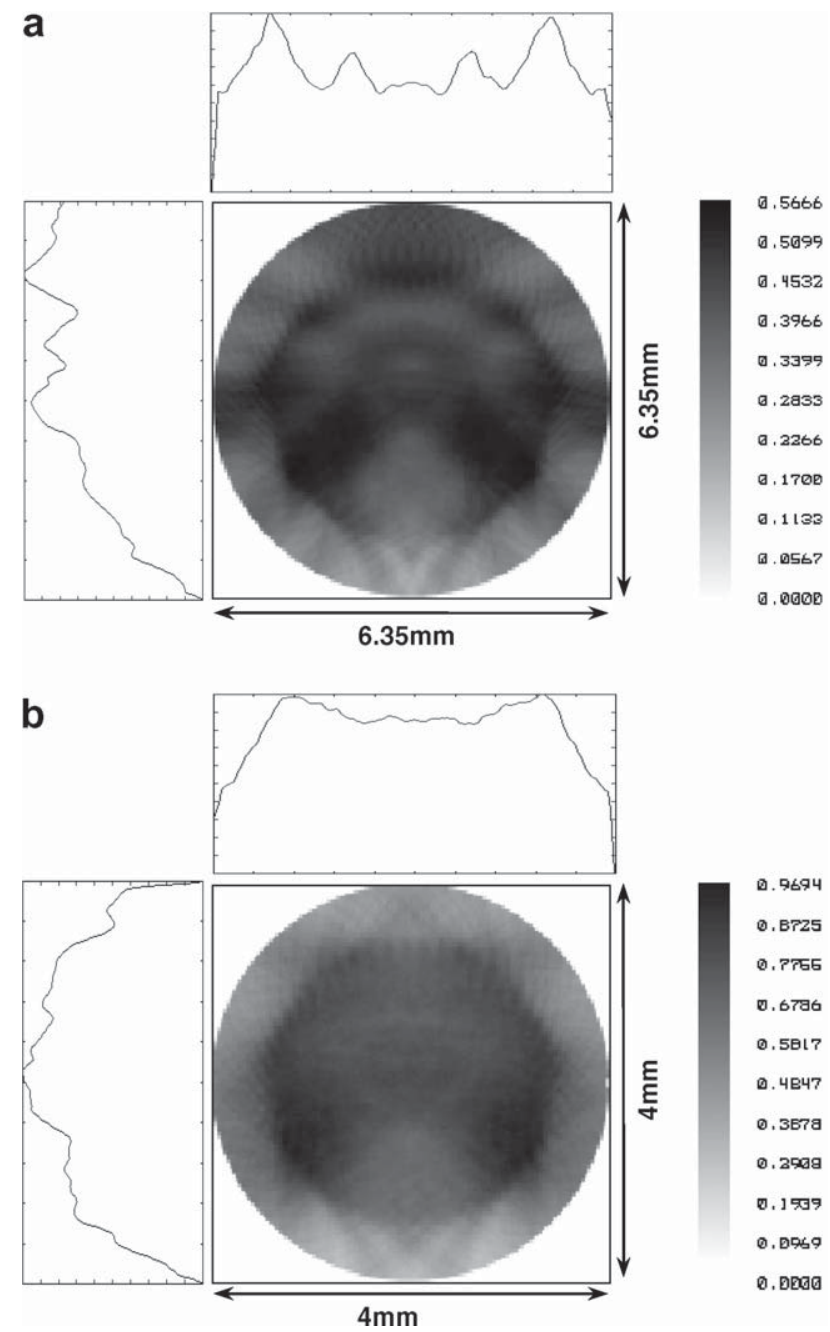

Fig. 9. Grey-scale absorption distribution within the 2D-CPC-CPC cavity pumped by the rectangular source of $80 \mathrm{~mm}$ width for (a) $6.35 \mathrm{~mm}$ diameter (b) $4.0 \mathrm{~mm}$ diameter $\mathrm{Nd}$ :YAG rods (vertical unit in $\mathrm{W} / \mathrm{mm}^{2}$ ).

tracing software. The values listed in Table 7 confirm the superiority of the EL type cavity over the CPC type cavity. Capable of concentrating more pump energy within the central core region, the 2D-CPC-EL cavity offers the same effectiveness as the 16-side-pumping approach. If the symmetrical pumping scheme is less than 16 sides ( 12 sides, for example) around the $80 \mathrm{~mm}$ perimeter, the 2D-CPC-EL cavity will then offer the best absorption efficiency for low order modes. In term of the total pump absorption by the rod, the 16-side-pumping approach is the most efficient while on the other hand, the CPC scheme offers the modest performances in every aspects.

The symmetric multi-side pumping scheme offers unrivalled symmetry for any asymmetric approaches. The angular absorption distribution analysis will therefore include only the 2D-CPC-EL and the 2D-CPC-CPC cavities. The absorption distribution within the laser medium is rather complex. Angular absorption analysis is hence carried out to evaluate angular non-uniformities. In ZEMAX ray-tracing analysis, the angular distribution at a specific 

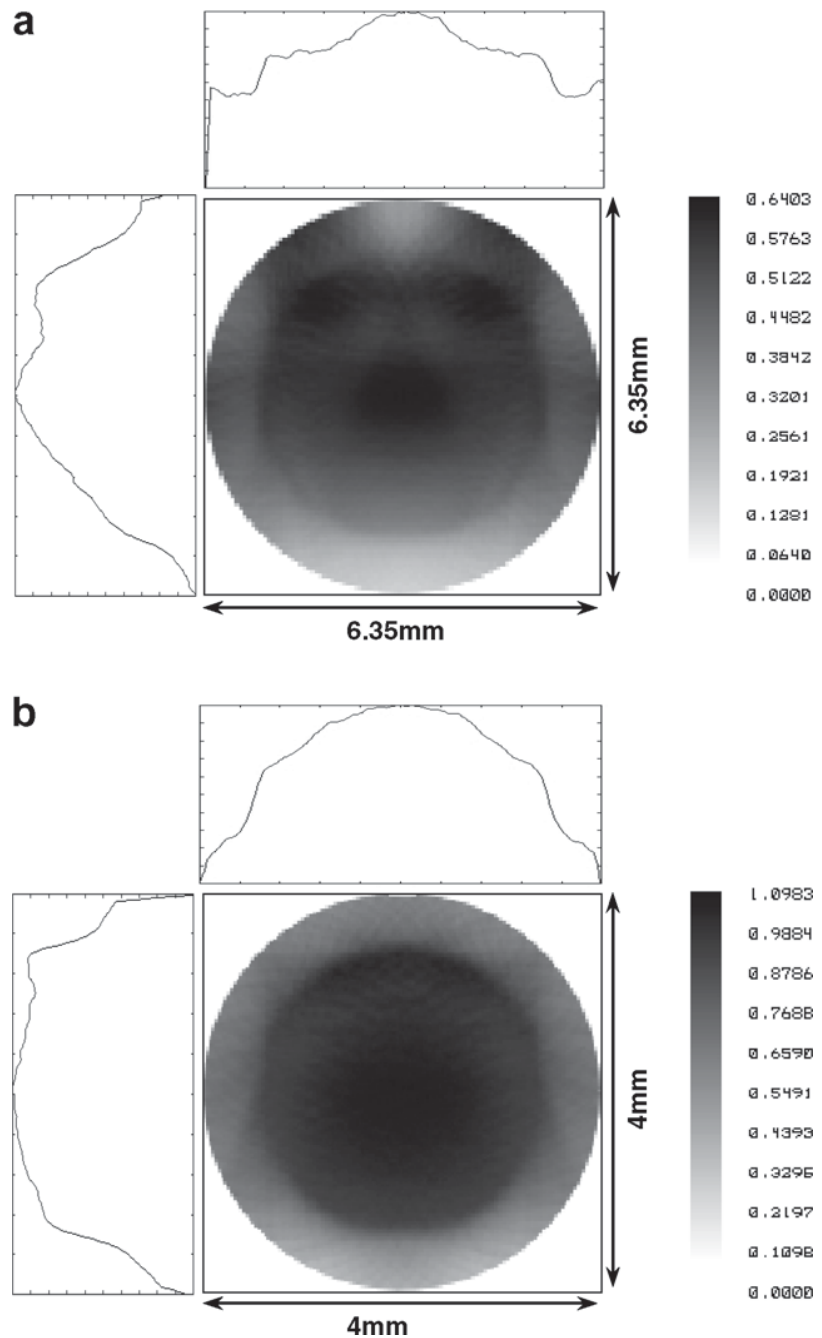

Fig. 10. Grey-scale absorption distribution within the 2D-CPC-EL cavity pumped by the rectangular source of $100 \mathrm{~mm}$ width for (a) $6.35 \mathrm{~mm}$ diameter (b) $4.0 \mathrm{~mm}$ diameter Nd:YAG rods (vertical unit in $\mathrm{W} / \mathrm{mm}^{2}$ ).

angle can be calculated by integrating the absorbed flux over all the zones distributed along that angle. The absorbed flux along the length of the rod is integrated axially. The radial integration starts from the centre of the laser medium and ends at a desired radius. The radius of $0.5 \mathrm{~mm}$ and $1.0 \mathrm{~mm}$ are chosen to analyse the angular non-uniformities within the central cylinders of $1.0 \mathrm{~mm}$ and $2.0 \mathrm{~mm}$ diameters respectively. This analysis is important for enhancing the production of both $\mathrm{TEM}_{00}$ and loworder laser modes. The radius of $3.175 \mathrm{~mm}$ and $2.0 \mathrm{~mm}$ are used to accomplish the angular non-uniformity analysis around the whole rods.

By analysing the absorbed flux/volume values in Figs. 6-11 and by examining the absorbed efficiency improvements in Tables 4-6, conclusion can be reached that there is a significant increase in the average absorbed flux levels for the angular absorption curves when the rods are pumped by the 2D-CPC-EL cavity. This absorbed flux level also increases with the reduction in the width of the 2-D ESDA pump source. To analyse only on the relative
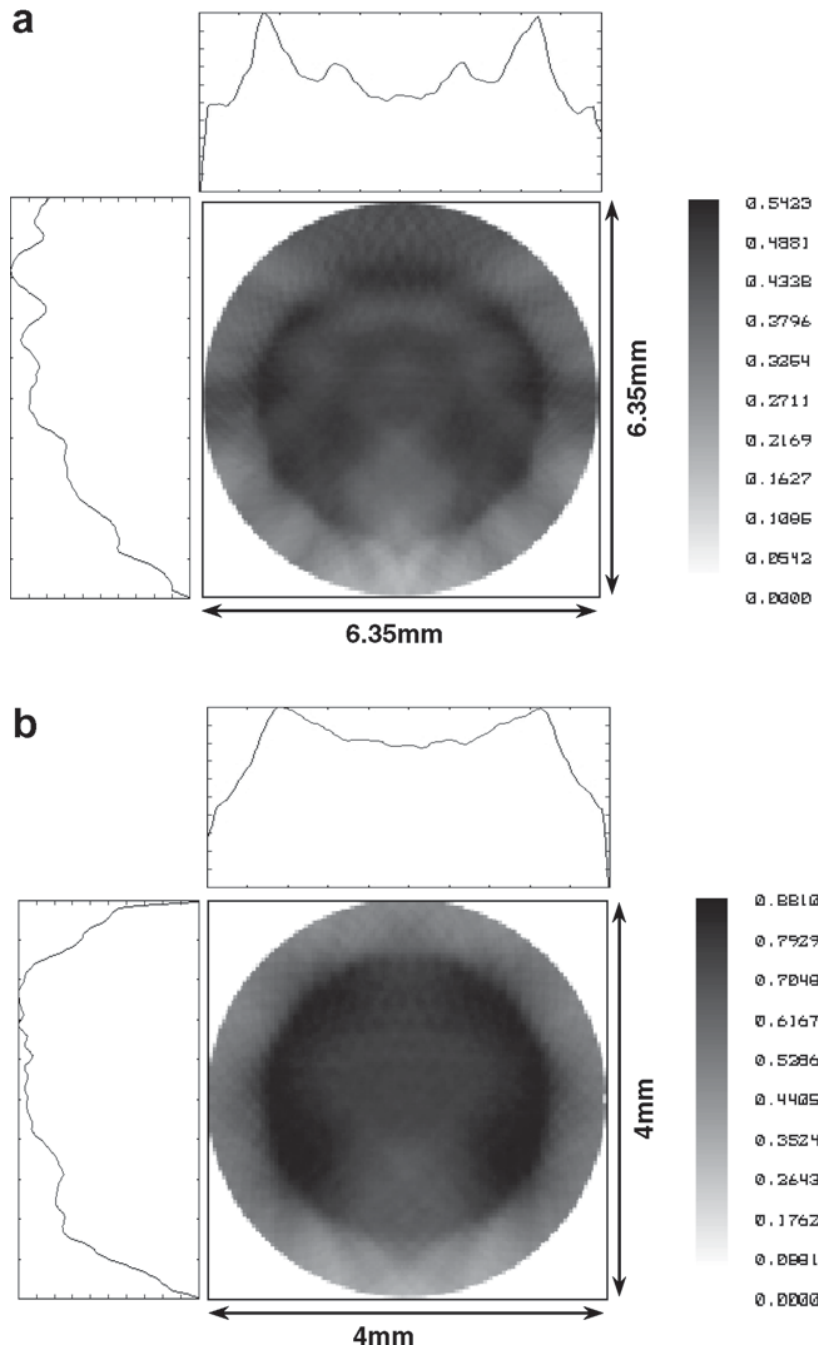

Fig. 11. Grey-scale absorption distribution within the 2D-CPC-CPC cavity pumped by the rectangular source of $100 \mathrm{~mm}$ width for (a) $6.35 \mathrm{~mm}$ diameter (b) $4.0 \mathrm{~mm}$ diameter $\mathrm{Nd}: \mathrm{YAG}$ rods (vertical unit in $\mathrm{W} / \mathrm{mm}^{2}$ ).

intensity variations of the curves themselves, all the absorbed flux values of each curve, integrated over the angles varying between $0^{\circ}$ and $360^{\circ}$, are normalized to unity. Depending on different pumping widths of $60 \mathrm{~mm}$, $80 \mathrm{~mm}$ or $100 \mathrm{~mm}$, the normalized angular absorption curves are given by Figs. 12-14 respectively. The horizontal axis is the angle in degrees measured anti-clockwise from the positive $x$ axis of the laser rod. When the laser rod is pumped by the 2D-CPC-EL cavity, the angular absorption distributions within the $1.0 \mathrm{~mm}$ and $2.0 \mathrm{~mm}$ cylindrical core volumes reveals a much less variations than those curves pumped by the 2D-CPC-CPC cavity. The angular absorption distributions around $4.0 \mathrm{~mm}$ and $6.35 \mathrm{~mm}$ crystals are also noticeably enhanced by 2D-CPC-EL cavity, resulting in more absorption uniformity. It is also worthwhile to mention the excellent angular absorption distribution of $4.0 \mathrm{~mm}$ rod within the 2D-CPC-EL cavity. Even though not as effective as it is for enhancing the angular uniformity for $4.0 \mathrm{~mm}$ crystal, the 2D-CPC-EL cavity still ensures a considerable improvement in the absorption dis- 
Table 4

Absorption efficiencies of laser rods pumped by the rectangular source of $60 \mathrm{~mm}$ width

\begin{tabular}{llll}
\hline $\begin{array}{l}\text { Rod } \\
\text { diameter } \\
(\mathrm{mm})\end{array}$ & $\begin{array}{l}\text { Absorption } \\
\text { efficiency }(\%)\end{array}$ & $\begin{array}{l}\text { Within } \varnothing 2 \mathrm{~mm} \\
\text { cylindrical volume } \\
(\%)\end{array}$ & $\begin{array}{l}\text { Within } \varnothing 1 \mathrm{~mm} \\
\text { cylindrical volume } \\
(\%)\end{array}$ \\
\hline EL 6.35 & 83.12 & 12.53 & 3.53 \\
CPC 6.35 & 72.40 & 7.54 & 2.03 \\
EL 4.0 & 62.41 & 20.43 & 5.77 \\
CPC 4.0 & 47.80 & 12.70 & 3.32 \\
\hline
\end{tabular}

Table 5

Absorption efficiencies of laser rods pumped by the rectangular source of $80 \mathrm{~mm}$ width

\begin{tabular}{llll}
\hline $\begin{array}{l}\text { Rod } \\
\text { diameter } \\
(\mathrm{mm})\end{array}$ & $\begin{array}{l}\text { Absorption } \\
\text { efficiency }(\%)\end{array}$ & $\begin{array}{l}\text { Within Ø2 mm } \\
\text { cylindrical volume } \\
(\%)\end{array}$ & $\begin{array}{l}\text { Within } \varnothing 1 \mathrm{~mm} \\
\text { cylindrical volume } \\
(\%)\end{array}$ \\
\hline EL 6.35 & 80.80 & 10.70 & 2.95 \\
CPC 6.35 & 72.40 & 7.64 & 2.04 \\
EL 4.0 & 57.34 & 17.80 & 4.89 \\
CPC 4.0 & 48.30 & 12.80 & 3.34 \\
\hline
\end{tabular}

Table 6

Absorption efficiencies of laser rods pumped by the rectangular source of $100 \mathrm{~mm}$ width

\begin{tabular}{llll}
\hline $\begin{array}{l}\text { Rod } \\
\text { diameter } \\
(\mathrm{mm})\end{array}$ & $\begin{array}{l}\text { Absorption } \\
\text { efficiency }(\%)\end{array}$ & $\begin{array}{l}\text { Within Ø2 mm } \\
\text { cylindrical volume } \\
(\%)\end{array}$ & $\begin{array}{l}\text { Within Ø1 mm } \\
\text { cylindrical volume } \\
(\%)\end{array}$ \\
\hline EL 6.35 & 77.42 & 9.19 & 2.45 \\
CPC 6.35 & 78.30 & 8.42 & 2.12 \\
EL 4.0 & 53.30 & 15.95 & 4.19 \\
CPC 4.0 & 45.03 & 13.80 & 3.46 \\
\hline
\end{tabular}

Table 7

Absorption efficiencies by three different types of cavities pumped by $L=80 \mathrm{~mm}$ for the Nd:YAG absorption coefficients of $10 \mathrm{~cm}^{-1}$ and $6 \mathrm{~cm}^{-1}$

\begin{tabular}{llll}
\hline $\begin{array}{l}\text { Rod } \\
\text { diameter } \\
(\mathrm{mm})\end{array}$ & $\begin{array}{l}\text { Absorption } \\
\text { efficiency }(\%)\end{array}$ & $\begin{array}{l}\text { Within Ø2 mm } \\
\text { cylindrical volume } \\
(\%)\end{array}$ & $\begin{array}{l}\text { Within Ø1 mm } \\
\text { cylindrical volume } \\
(\%)\end{array}$ \\
\hline $\begin{array}{l}\text { Abs. } 10 \mathrm{~cm}^{-1} \\
\begin{array}{l}\text { 16-side } \\
\quad 9.35\end{array}\end{array}$ & 93.82 & 4.59 & 1.12 \\
EL 6.35 & 87.30 & 4.57 & 1.15 \\
CPC 6.35 & 79.30 & 3.18 & 0.77 \\
Abs. $6 \mathrm{~cm}^{-1}$ & & & \\
$\begin{array}{l}\text { 16-side } \\
\quad 9.35\end{array}$ & 91.53 & 9.00 & 2.33 \\
EL 6.35 & 85.62 & 8.93 & 2.42 \\
CPC 6.35 & 77.40 & 6.23 & 1.62 \\
\hline
\end{tabular}

tribution for $6.35 \mathrm{~mm}$ crystal. Due to the less flux fluctuations, there is more pump symmetry for the 2D-CPC-EL cavity. The 2D-CPC-CPC cavity presents the modest pump symmetry whereas the superb pump symmetry of conventional multi-side pumping scheme shown in Fig. 2 is very difficult to challenge for any of the asymmetric approaches.

\section{Comparisons between three types of pumping cavities}

Through the ray-tracing analysis of three different types of pump cavities, some interesting features are found. For the same rectangular light emitting surface of $80 \mathrm{~mm}$ width and $100 \mathrm{~mm}$ length, the laser rod can either be symmetrically pumped by many narrow vertical diode stacks distributed along a cylindrical surface with $80 \mathrm{~mm}$ perimeter or asymmetrically pumped from only one side by a twodimensional pump source of $80 \mathrm{~mm}$ width, through either the 2D-CPC-EL or 2D-CPC-EL cavities. By analysing Figs. 2, 8 and 9, Tables 1 and 5, some conclusions can be reached:
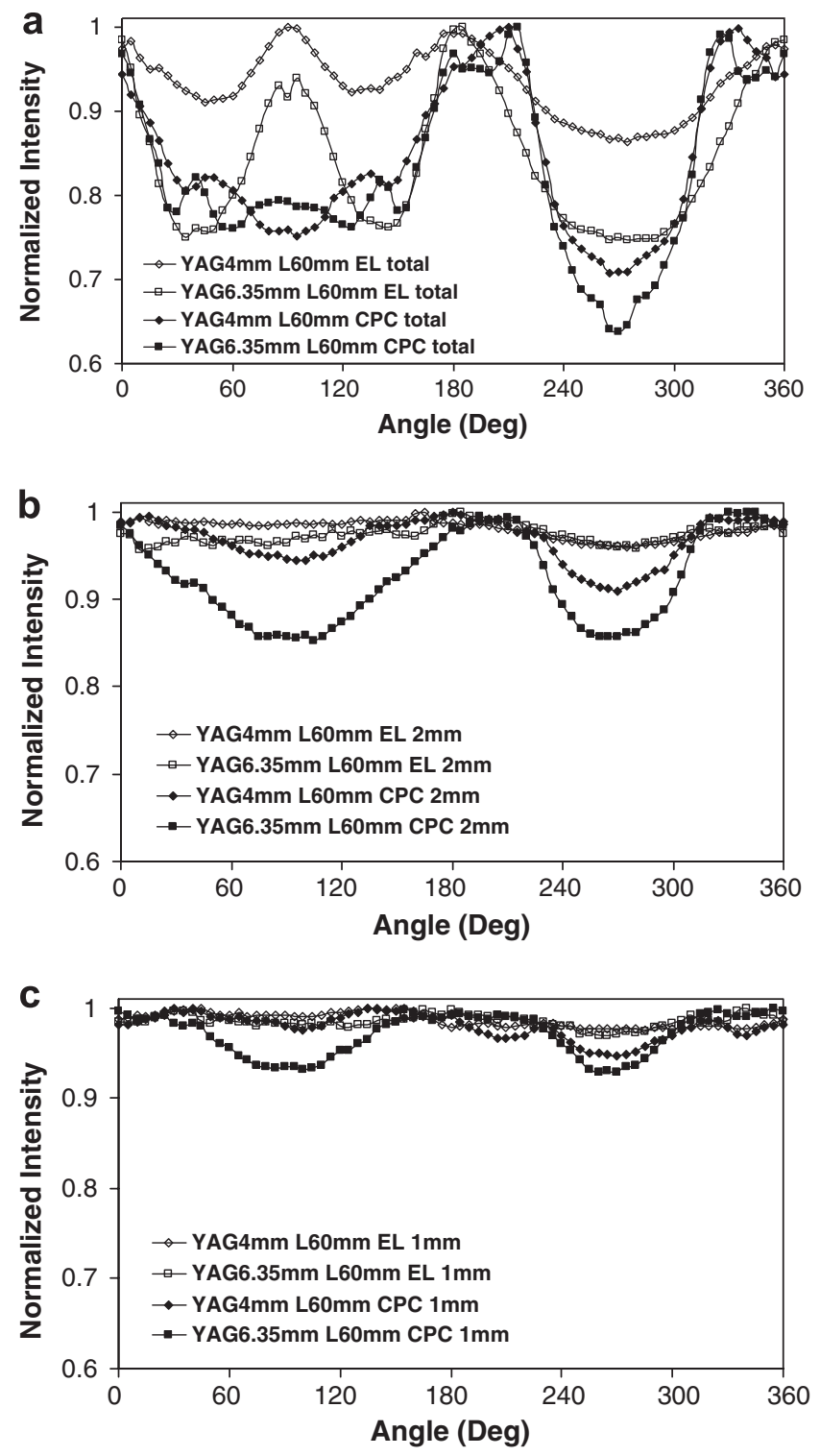

Fig. 12. Angular distribution of the absorbed pump power normalized to unity is calculated both for $6.35 \mathrm{~mm}$ and $4 \mathrm{~mm}$ diameter rods inside a cylindrical volume of (a) total crystal diameters, (b) $2 \mathrm{~mm}$ diameter (c) $1 \mathrm{~mm}$ diameter. The curves for both the 2D-CPC-EL and the $2 \mathrm{D}-\mathrm{CPC}-$ CPC cavities are shown for the pumping width of $60 \mathrm{~mm}$. The horizontal axis is the angle in degrees measured clockwise from the positive $x$ axis of the rod in Fig. 5. Average absorption coefficient of $4 \mathrm{~cm}^{-1}$ is considered. 

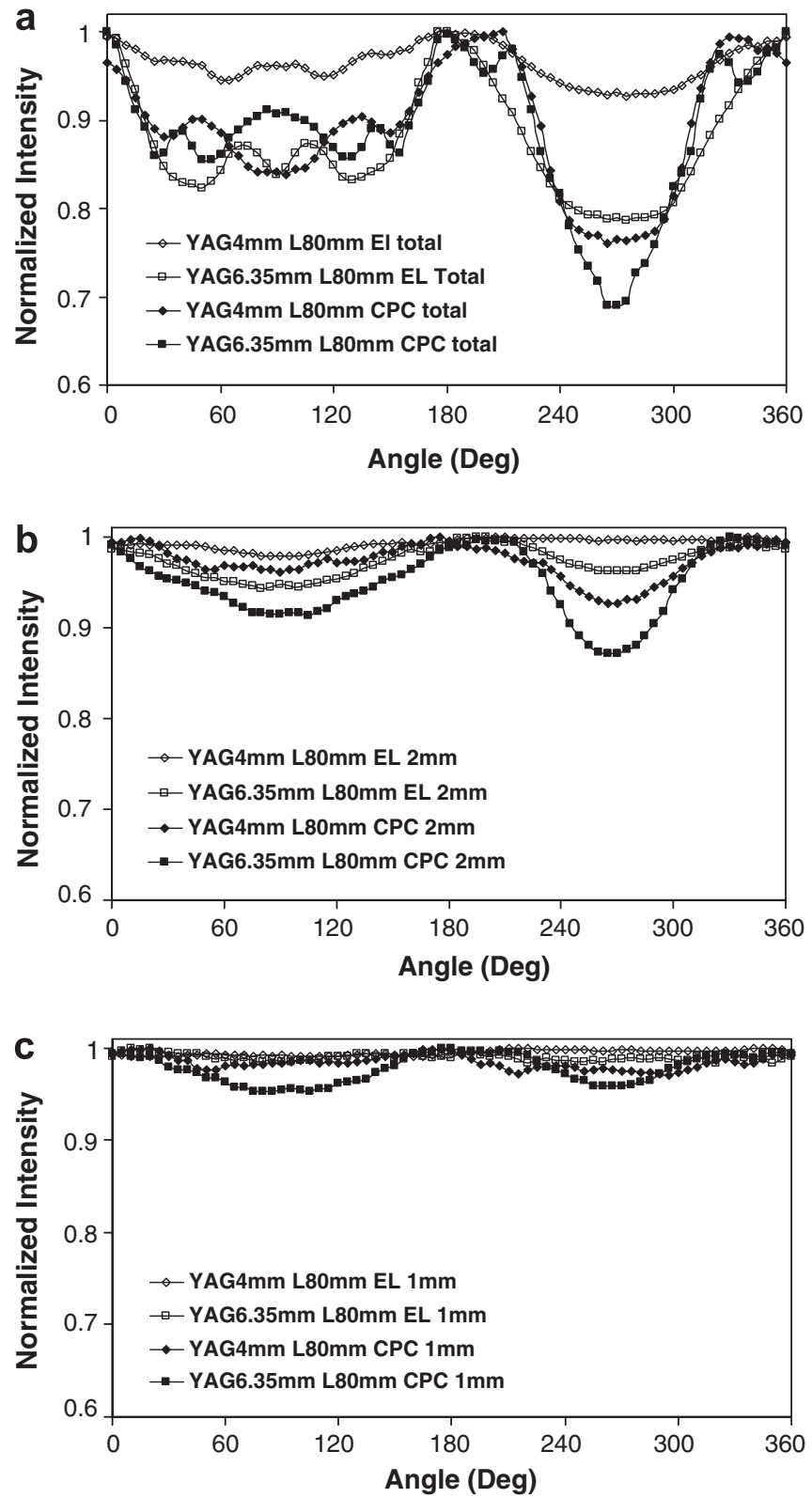

Fig. 13. Angular distribution of the absorbed pump power normalized to unity is calculated both for $6.35 \mathrm{~mm}$ and $4 \mathrm{~mm}$ diameter rods inside a cylindrical volume of (a) total crystal diameters, (b) $2 \mathrm{~mm}$ diameter (c) $1 \mathrm{~mm}$ diameter. The curves for both the 2D-CPC-EL and the 2DCPC-CPC cavities are shown for the pumping width of $80 \mathrm{~mm}$. The horizontal axis is the angle in degrees measured clockwise from the positive $x$ axis of the rod in Fig. 5. Average absorption coefficient of $4 \mathrm{~cm}^{-1}$ is considered.

1. The symmetrical and direct pumping scheme offers the best absorption efficiency and excellent beam quality for the side pumping of at least 16 sides along the $80 \mathrm{~mm}$ perimeter. Any attempt to reduce the number of sides will degrade the absorption efficiency and distribution. The practical considerations such as the commercial availability of the narrow vertical diode stack with only several millimetres width and the difficulty in both mounting and wavelength tuning of many diode stacks need, in our opinion, to be balanced with the
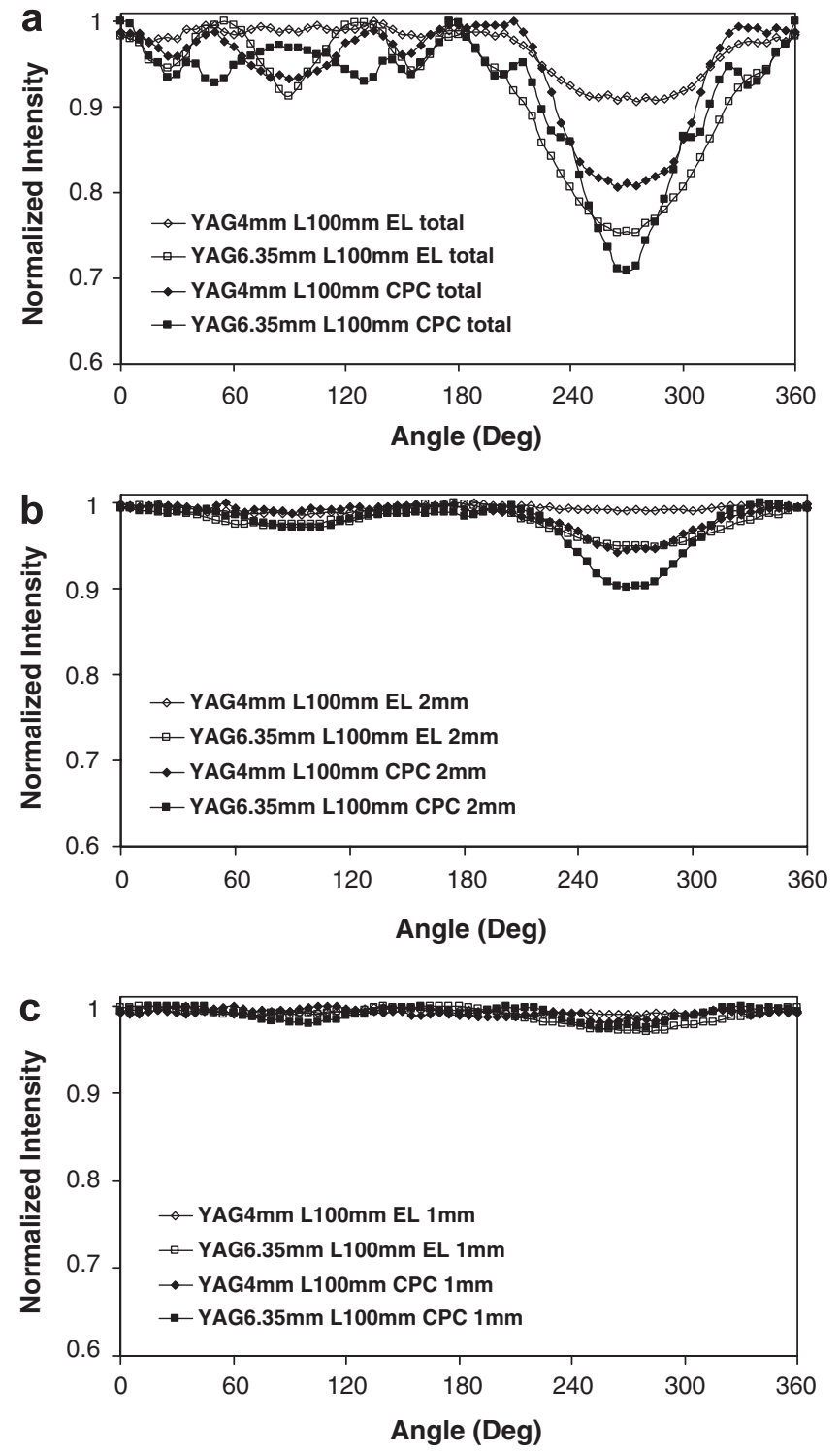

Fig. 14. Angular distribution of the absorbed pump power normalized to unity is calculated both for $6.35 \mathrm{~mm}$ and $4 \mathrm{~mm}$ diameter rods inside a cylindrical volume of (a) total crystal diameters, (b) $2 \mathrm{~mm}$ diameter (c) $1 \mathrm{~mm}$ diameter. The curves for both the 2D-CPC-EL and the 2DCPC-CPC cavities are shown for the pumping width of $100 \mathrm{~mm}$. The horizontal axis is the angle in degrees measured clockwise from the positive $x$ axis of the rod in Fig. 5. Average absorption coefficient of $4 \mathrm{~cm}^{-1}$ is considered.

necessity of highly symmetric gain distribution when a high-average-power diode-pumped solid-state laser is to be designed.

2. The 2D-CPC-EL cavity addresses the asymmetrical pumping problem by making a full use of the commercially available two-dimensional micro-lens-free high power diode stacks in high-average-power regime. The pump radiation absorption efficiency within the central core volumes has reached the same level as the symmetrical scheme, as indicated by Tables 1,5 and 7 . The absorption distributions given by Figs. 6, 8 and 10, are satisfactory for many solid-state laser applications. Considering the drawbacks of the symmetrical multi-side 
pumping scheme discussed in Section 2, the 2D-CPCEL cavity has hence the same effectiveness as the symmetric approach. The absorption efficiency indicates that the two-dimensional truncated elliptical cavity with intervening optics is also beneficial for the production of $\mathrm{TEM}_{00}$ laser beam.

3. Contrary to the 2D-CPC-CPC pump scheme, where any attempt to include flow tube means redesigning the cavity, the flow tube with water can easily be included into the 2D-CPC-EL cavity. The 2D-CPC-CPC cavity can neither compete with the symmetric multi-side pumping scheme nor with the asymmetric 2D-CPC-EL approaches. Both the absorption efficiency and gain distribution are not qualified for the efficient production of high quality laser beam.

4. Considering the possible application to Sun-pumped lasers, where broadband solar radiation is highly concentrated to achieve the infrared laser emission, the ray-tracing analysis for the 2D-CPC-EL cavity with uncoated optical components is carried out, revealing the similar absorption efficiency and distribution performances. There is however a several percent reduction in all the absorption efficiencies.

\section{Conclusions}

By adopting the 2D-CPC-EL pump cavity with cylindrical intervening optics, what we believe to be a novel scheme for achieving a strong pump absorption within the small centred volume of the Nd:YAG laser rod is proposed. The pump radiation from a large area two-dimensional diode stack is concentrated by the first-stage 2D-CPC to the rectangular entry aperture of the second-stage truncated 2D-EL pump cavity with a cylindrical intervening optics. The concentrated pump radiation from the intervening optics is then efficiently coupled to the laser rod mounted within the cooling jacket. Depending on the width of the rectangular pump source, the optimized rod mounting position is decided by the non-sequential raytracing analysis. The image formation properties of both the elliptical cavity and the intervening optics play important roles in obtaining the highly concentrated pump radi- ation within the rod as compared with the performance of the 2D-CPC-CPC cavity. The absorption efficiencies and absorption distributions of both the 2D-CPC-EL and the 2D-CPC-CPC pump cavities are analysed, favouring the elliptical type. The rectangular pump source and the $4 \mathrm{~mm}$ diameter rod are found to be suitable choices for reaching good quality absorption distribution. Capable of coupling efficiently the pump radiation from the commercially available two-dimensional micro-lens-free diode stacks, this asymmetric side pumping scheme offers an effective alternative to conventional symmetric pumping scheme in high-average-power regime. The proposed pump scheme can also hopefully be applied to the researches on solar lasers.

\section{References}

[1] F. Brioschi, E. Nava, G.C. Reali, IEEE J. Quantum. Electron. 28 (4) (1992) 1070.

[2] T.Y. Fan, A. Sanchez, IEEE J. Quantum. Electron. 26 (1990) 311.

[3] J.J. Chang, E.P. Dragon, I.L. Bass, in: Conference on Lasers and Electro-Optics (CLEO/US)1998 OSA Technical Digests, vol. 6, Optical Society of America, Washington, DC, 1998, p. CPD2-2.

[4] S. Fujikawa, K. Furuta, K. Yasui, Opt. Lett. 26 (9) (2001) 602.

[5] H. Moon, J. Yi, J. Han, B. Cha, J. Lee, Appl. Opt. 38 (9) (1999) 1772.

[6] T. Brand, Opt. Lett. 20 (17) (1995) 1776.

[7] R.J. Beach, Appl. Opt. 35 (12) (1996) 2000.

[8] E.C. Honea, R.J. Beach, S.C. Mitchell, P.V. Avizonis, Opt. Lett. 24 (1999) 154.

[9] D. Golla, S. Knoke, W. Schöne, G. Ernst, M. Bode, A. Tünnermann, H. Welling, Opt. Lett. 20 (1995) 1148.

[10] R.J. Koshel, I.A. Walmsley, Opt. Eng. 43 (7) (2004) 1511.

[11] R.J. Koshel, I.A. Walmsley, Appl. Opt. 32 (9) (1993) 1517.

[12] W.T. Welford, R. Winston, High Collection Nonimaging Optics, Academic Press, New York, 1989.

[13] M. Lando, J. Kagan, B. Linyekin, V. Dorbrusin, Opt. Commun. 222 (2003) 371.

[14] K. Kamiryo, T. Kano, H. Matsuzawa, Jpn J Appl Phys 5 (12) (1966) 1217.

[15] S.B. Schuldt, R.L. Aagard, Appl. Opt. 2 (5) (1963) 509.

[16] C. Bowness, Appl. Opt. 4 (1) (1965) 103.

[17] P.H. Bernardes, D. Liang, Appl. Opt. 45 (16) (2006) 3811.

[18] W. Koechner, Solid-State Laser Engineering, fourth ed., Optical Sciences, vol. 1, Springer-Verlag, Berlin, Germany, 1992 (Chapter 6, p. 378).

[19] R. Winston, Appl. Opt. 17 (9) (1978) 1668. 